CD3 and HER2. Attached to the core, two unstructured polypeptide masks (XTEN) sterically reduce target engagement and extend T1/2. Protease cleavage sites at the base of the XTEN masks enable proteolytic activation of XPATs in the tumor microenvironment, unleashing a potent TCE with short T1/2, further improving the TI. HER2-XPAT, a tumor protease-activatable prodrug with wide safety margins, can co-opt T-cells regardless of antigenic specificity to induce T-cell killing of HER2+ tumors.

Methods Preclinical studies were conducted to characterize the activity of HER2-XPAT, HER2-PAT (cleaved XPAT), and HER2-NonClv (a non-cleavable XPAT) for cytotoxicity in vitro, for anti-tumor efficacy in xenograft models, and for safety in NHPs.

Results HER2-PAT demonstrated potent in vitro T-cell cytotoxicity (EC50 1-2pM) and target-dependent T-cell activation and cytokine production by hPBMCs. HER2-XPAT provided up to 14,000 -fold protection against killing of HER2 tumor cells and no cytotoxicity against cardiomyocytes up to $1 \mathrm{uM}$. In vivo, HER2-XPAT induced complete tumor regressions in BT-474 tumors with equimolar dosing to HER2-PAT, whereas HER2-NonClv had no efficacy, supporting requirement of protease cleavage for T-cell activity. In NHP, HER2-XPAT has been dose-escalated safely up to $42 \mathrm{mg} / \mathrm{kg}$ (MTD). HER2XPAT demonstrated early T-cell margination at $2 \mathrm{mg} / \mathrm{kg}$ but largely spared CRS, cytokine production, and tissue toxicity up to $42 \mathrm{mg} / \mathrm{kg}$. PK profiles of HER2-XPAT and HER2NonClv were comparable, consistent with ex vivo stability for cleavage when incubated in cancer pts plasma for 7 days at $37^{\circ} \mathrm{C}$. HER2-PAT by continuous infusion induced lethal CRS and cytokine spikes at $0.3 \mathrm{mg} / \mathrm{kg} / \mathrm{d}$ but was tolerated at 0.25 $\mathrm{mg} / \mathrm{kg} / \mathrm{d}$, providing HER2-XPAT with $>1300$-fold protection in tolerability vs. HER2-PAT, $>4$ logs over cytotoxicity EC50s for HER2 cell lines, and a 20-fold safety margin over the dose required for pharmacodynamic activity.

Conclusions HER2-XPAT is a potent prodrug TCE with no CRS and a wide TI based on NHPs. With XTEN's clinical data demonstrating low immunogenicity, the XPATs are a promising solution. IND studies are ongoing. Additional PK/ PD, cytokines, safety, and efficacy data will be presented.

http://dx.doi.org/10.1136/jitc-2020-SITC2020.0613

\section{CO-STIMULATION VIA PD1-41BB CHIMERIC SWITCH RECEPTOR ENHANCES FUNCTION OF TCR-T CELLS IN AN IMMUNE-SUPPRESSIVE MILIEU AND UNDER CHRONIC ANTIGEN STIMULATION}

${ }^{1}$ Melanie Salvermoser, ${ }^{1}$ Maria Gerget, ${ }^{1}$ Franziska Hasselmann, ${ }^{2}$ Elfriede Noessner ${ }^{1}$ Christian Ellinger, ${ }^{1}$ Monika Braun, ${ }^{3}$ Dolores Schendel, ${ }^{1}$ Daniel Sommermeyer, ${ }^{1}$ Nadja Sailer*. 'Medigene Immunotherapies GmbH, Planegg-Martinsried, Germany; ${ }^{2}$ Helmholtz Zentrum München, Munich, Germany; ${ }^{3}$ Medigene AG, Planegg-Martinsried, Germany

Background The immunosuppressive tumor microenvironment (TME) of solid tumors negatively influences the efficacy and fitness of tumor-specific $T$ cells and can render them nonfunctional. In this repressive tumor milieu, expression of inhibitory immune checkpoint molecules and cytokines as well as deprivation of essential metabolic factors contribute to $\mathrm{T}$ cell exhaustion and reduced $\mathrm{T}$ cell infiltration. Due to these harsh conditions found in the TME of solid tumors, successful treatment of non-hematological cancer indications with $\mathrm{T}$ cellbased immunotherapies remains challenging. New strategies are required to equip therapeutic tumor-specific $\mathrm{T}$ cells with the necessary traits to overcome inhibitory signals in the TME and increase $\mathrm{T}$ cell persistence in an environment lacking essential metabolic nutrients, like oxygen or glucose. To enhance the clinical efficacy of TCR-T cells in treatment of solid tumors, we generated a chimeric receptor that combines the co-stimulatory domain of $4-1 \mathrm{BB}$ with the extracellular domain of PD-1. Expression of this chimeric PD1-41BB switch receptor in TCR-T cells should reverse the inhibitory signal induced by the PD-1/PD-L1 interaction and provide additional co-stimulation to increase functionality and persistence.

Methods Using 2D and 3D in vitro model systems we mimic immunosuppressive conditions in the TME of solid tumors, including low glucose and high TGFbeta levels as well as repeated tumor cell challenge. We evaluate the ability of the chimeric PD1-41BB switch receptor to enhance TCR-T cell activity and functionality under these repressive conditions.

Results Our results demonstrate that TCR-T cells expressing the chimeric PD1-41BB switch receptor show an increased capacity to recognize and kill tumor cells during chronic stimulation with antigen. The enhanced functionality of PD141BB-TCR-T cells allows them to eradicate tumor cells even in the presence of additional immunosuppressive factors, including nutrient starvation and expression of inhibitory PD-L1 checkpoint molecules. Furthermore, PD1-41BB-expressing TCR-T cells show a higher persistency and proliferation rate in these challenging co-culture model systems.

Conclusions Equipping therapeutic $\mathrm{T}$ cells with the chimeric PD1-41BB switch receptor enhances $\mathrm{T}$ cell functionality under immunosuppressive conditions and counteracts checkpointmediated dysfunction. For the treatment of PD-L1-poitive malignancies, expression of PD1-41BB by TCR-T cells has the potential to greatly improve the targeting of solid tumors using $\mathrm{T}$ cell-based immunotherapies. These preclinical studies support our approach to enhance the clinical efficacy of TCR$\mathrm{T}$ therapies of solid tumors using the chimeric PD1-41BB switch receptor. Subsequent in vivo studies and safety evaluations will pave the way for clinical use of PD1-41BB in adoptive $\mathrm{T}$ cell therapy.

http://dx.doi.org/10.1136/jitc-2020-SITC2020.0614

\section{TARGETED IMMUNE CELL ACTIVATION BY SYSTEMIC DELIVERY OF TOLL-LIKE RECEPTOR 9 AGONIST ANTIBODY CONJUGATES INDUCE POTENT ANTI-TUMOR IMMUNITY}

${ }^{1}$ Ons Harrabi*, 'Amy Chen, 'Emma Sangalang, 'Laura Doyle, 'Danielle Fontaine, ${ }^{1}$ Bora Han, ${ }^{2}$ Curt Bradshaw, ${ }^{3}$ Jaume Pons, ${ }^{1}$ Janet Sim, ${ }^{1}$ Tracy Kuo, ${ }^{1}$ Hong Wan. ${ }^{1}$ Tallac Therapeutics, Burlingame, CA, USA; ${ }^{2}$ Arrowhead Pharmaceuticals, Pasadena, CA, USA; ${ }^{3}$ ALX Oncology, Inc., Burlingame, CA, USA

Background Toll-like receptor (TLR) pathways play a crucial role in mounting potent innate immune responses against invading pathogens, as well as the subsequent engagement of adaptive immunity. Innate immune activation via the TLR9 pathway has potential for treating cancer as demonstrated clinically with TLR9 agonists administered intra-tumorally in melanoma patients. ${ }^{1}$ We developed a novel toll-like receptor agonist antibody conjugate (TRAAC) platform to systemically deliver a differentiated, targeted TLR9 agonist (T-CpG) for immune activation. The activation of TLR9 pathways can be directed systemically towards specific immune cell populations and tumor microenvironment via antibodies binding to various 